UDC 510.6

\title{
On Some Universal Propositional Proof Systems for Many-Valued Logic
}

\author{
Artur A. Khamisyan \\ Yerevan State University \\ e-mail: khamisyam@gmail.com
}

\begin{abstract}
Some uniform Hilbert-like propositional proof system is suggested for all versions of many-valued logic to apply them to 3 versions of 3 -valued logic, two of which have only one designated value, and the last one has two designated values.

Keywords: Many-valued logic, Hilbert-like proof system, Uniform propositional proof system.
\end{abstract}

\section{Introduction}

It is known that many-valued logic (MVL) as a separate subject was first created and developed by Łukasiewicz [1]. His intention was to use a third, additional truth value for "possible" (or "unknown"). In the meantime, many interesting applications of MVL were found in such fields as logic, mathematics, hardware design, artificial intelligence and some other areas of soft information technologies, therefore investigations in the area of many-valued logic are very actual. The main theoretical results concern several properties of formal systems, which can present different versions of MVL and, in particular, issues on logical completeness of such systems. The completeness of some types of proof systems is proved by hard, many-stepped operations of immersion into two-valued logic, and for the other systems itis proved by reducing tothe completeness of the first type. The generalization of Kalmar's proof of deducibility for two-valued tautologies in classical propositional logic [2] enables us to suggest 1) a new method of proving the completeness of a propositional proof system (PPS) of some well-known MVL such that it is essentially simpler than other known proofs of completeness and can be easily modified even into a proof of completeness for fuzzy logic as well, 2) a method for defining of many traditional variants of PPS for MVL, the completeness of which is easily proved directly, without the usual immersion into two-valued logic. Several new PPS for two versions of MVL are introduced in [3-7]. The current research refers to the problem of constructing some uniform Hilbert-like PPS for all possible versions of MVL. 


\section{Main Definitions of k-Valued Logics}

Here we give the main notions and notations for different versions of MVL.

Let $E_{k}$ be the set $\left\{0, \frac{1}{\mathrm{k}-1}, \ldots, \frac{\mathrm{k}-2}{\mathrm{k}-1}, 1\right\}$. We use the well-known notions of a propositional formula, which, as usual, are defined from propositional variables with values from $E_{k}$, (may be also propositional constants), parentheses (,), and logical connectives \& , $, \supset, \neg$, each of which can be defined by a different mode. Additionally, we introduce several variants of the exponential function $\boldsymbol{p}$.We use the well-known notion of a propositional formula and introduce an additional notion of a formula: for each formulas $\mathrm{A}$ and $\mathrm{B}$, the expression $\boldsymbol{A}^{\boldsymbol{B}}$ is also a formula.

In the considered logics, only 1 or each of the values $\frac{1}{2} \leq \frac{i}{\mathrm{k}-1} \leq 1$ can be fixed as designated values.

Definitions of main logical functions are:

$\boldsymbol{p} \vee \boldsymbol{q}=\max (p, q)(1)$ disjunction,

$\boldsymbol{p} \vee \boldsymbol{q}=\min (p+q, 1)(2)$ disjunction,

$\boldsymbol{p} \& q=\min (p, q) \quad$ (1) conjunction,

$\boldsymbol{p} \& q=p q(\bmod k) /(k-1) \quad$ (2) conjunction

For implication, we have the following two versions:

$\boldsymbol{p} \supset \boldsymbol{q}=\left\{\begin{array}{ll}1, & \text { for } p \leq q \\ 1-p+q, & \text { for } p>q\end{array} \quad\right.$ (1) Łukasiewicz's implication or

$\mathbf{p} \supset \boldsymbol{q}=\left\{\begin{array}{llll}1, & \text { for } & p \leq q \\ q, & \text { for } & p>q\end{array} \quad\right.$ (2) Gödel's implication

And for negation, there are also two versions:

$\neg \boldsymbol{p}=1-p \quad$ (1) Lukasiewicz's negation or

$\neg \boldsymbol{p}=((k-1) p+1)(\bmod k) /(k-1) \quad(2)$ cyclically permuting negation.

Sometimes we can use the notation $\overline{\boldsymbol{p}}$ instead of $\neg \boldsymbol{p}$.

For the propositional variable $\boldsymbol{p}$ and $\boldsymbol{\delta}=\frac{i}{\mathrm{k}-1}(0 \leq i \leq k-1)$, we additionally define "exponent" functions:

$\boldsymbol{p}$ as $(p \supset \delta) \&(\delta \supset p)$ with (1) implication $\quad$ (1) exponent,

$\boldsymbol{p}$ as $p$ with $(k-1)-i(2)$ negations.

(2) exponent.

Note that both (1) exponent and (2) exponent are not new logical functions.

If we fix " 1 " (each of the values $\frac{1}{2} \leq \frac{i}{\mathrm{k}-1} \leq 1$ ) as a designated value, then the formula $\varphi$ with variables $p_{1}, p_{2}, \ldots p_{n}$ is called a $\boldsymbol{k}$-tautology if for every $\tilde{\delta}=\left(\delta_{1}, \delta_{2}, \ldots, \delta_{n}\right) \in E_{k}^{n}$ assigning $\delta_{j}(1 \leq j \leq n)$ to each $p_{j}$ gives the value 1 (or some value $\frac{1}{2} \leq \frac{i}{\mathrm{k}-1} \leq 1$ ) of $\varphi$.

\section{Uniform Hilbert-Like Propositional Proof System and Its Properties}

Here we suggest some uniform Hilbert-like PPS for all possible versions of MVL. For all formulas $A, B, C$ of MVL, each $\sigma_{1}, \sigma_{2}$ from the set $E_{k}$ and for $* \in\{\&, \vee, \supset\}$ the following 
formulas are axiom schemes of some PPS version:

$$
\begin{aligned}
& \text { 1. } A \supset(B \supset A) \\
& \text { 2. }(A \supset B) \supset\left((A \supset(B \supset C)) \supset\left(A \supset C^{\varphi_{\text {m.p. }}(B, C)}\right)\right) \\
& \text { 3. } A^{\sigma} \supset(\neg A)^{\varphi_{\neg}(A, \sigma)} \\
& \text { 4.-6. } A^{\sigma_{1}} \supset\left(B^{\sigma_{2}} \supset(A * B)^{\varphi_{*}\left(A, B, \sigma_{1}, \sigma_{2}\right)}\right) \\
& \text { 7. } A^{\sigma_{1}} \supset\left(B^{\sigma_{2}} \supset\left(A^{B}\right)^{\varphi_{\exp }\left(A, B, \sigma_{1}, \sigma_{2}\right)}\right) \\
& \text { 8. }\left(A^{1} \supset B\right) \supset\left(\left(A^{k-1 / k-2} \supset B\right) \supset \cdots \supset\left(\left(A^{\frac{1}{k-1}} \supset B\right) \supset\left(\left(A^{0} \supset B\right) \supset B\right)\right) \ldots\right) \text {, }
\end{aligned}
$$

where many-valued functions $\varphi_{\neg}(A, \sigma), \varphi_{*}\left(A, B, \sigma_{1}, \sigma_{2}\right), \varphi_{\exp }\left(A, B, \sigma_{1}, \sigma_{2}\right)$ must be defined individually for each version of MVL, such that axioms 3-7 will be a $k$-tautology in this version.

Inference rule is a modification of modus ponens (m.m.p.) $\frac{A, A \supset B}{B^{\varphi_{\text {m.p. }}(A, B)}}$, where many-valued functions $\varphi_{\text {m.p. }}(A, B)$ must be defined individually for each version of MVL, such that the formula $A \supset\left((A \supset B) \supset B^{\varphi_{\text {m.p. }}(A, B)}\right)$ will also be a $k$-tautology in this version.

We use the well-known notions of proof and proof from premises. For all defined systems, we first prove the well known deduction theorem using the first two axiom schemes, and then the generalization of Kalmar's proof of deducibility for two valued tautologies inside the classical propositional logic gives us a possibility to directly prove completeness for the mentioned proof systems, without the usual loading into two-valued logic.

Let $P k$ be one of the defined PPS for some version of MVL. The following statement can be proved as usual.

Deduction theorem. Let $\Gamma$ be a set of some formulas and $A$ and $B$ be some formulas. If the formula $B$ is derived in the system $P k$ from the premises $\Gamma$ and $A\left(\Gamma, A \vdash_{P k} B\right)$, then the formula $A \supset B$ is derived in the system $P k$ from the premises $\Gamma\left(\Gamma \vdash_{P k} A \supset B\right)$.

Lemma 1: Let $P=\left\{p_{1}, p_{2}, \ldots, p_{n}\right\}$ be the set of all variables of any formula $A$, then for every $\tilde{\delta}=$ $\left(\delta_{1}, \delta_{2}, \ldots, \delta_{n}\right) \in E_{3}^{n}$

$$
p_{1}^{\delta_{1}}, p_{2}^{\delta_{2}}, \ldots, p_{n}^{\delta_{n}} \vdash_{C N 3} A^{A\left(\delta_{1}, \delta_{2}, \ldots, \delta_{n}\right)} .
$$

To simplify the proof, we demonstrate them only for $P 3$ logic.

Proof is given by induction on number $n$ of logical connectives in the formula A. For $n=1$ we have:

$$
\begin{aligned}
& \text { by } \delta=0 \quad p^{0} \vdash p^{0}, \\
& \text { by } \delta=1 / 2 p^{1 / 2} \vdash p^{1 / 2} \\
& \text { and by } \delta=1 \quad p^{1} \vdash p^{1} \text {. }
\end{aligned}
$$

Suppose that the statement is valid for the number of logical connectives $<\mathrm{n}$. If the number of logical connectives is $\mathrm{n}$, then the formula $A$ can be in one of the following forms:

1. $A=A_{1} * A_{2}$, where $* \in\{\&, \mathrm{~V}, \supset\}$,

2. $A=A_{1}^{A_{2}}$,

3. $A=\neg A_{1}$.

For the case 1. $A_{1}(\tilde{\delta})=\sigma_{1}, A_{2}(\tilde{\delta})=\sigma_{2}=>A(\tilde{\delta})=\sigma_{1} * \sigma_{2}$ 
By induction hypothesis

$$
\begin{aligned}
& p_{1}^{\delta_{1}}, p_{2}^{\delta_{2}}, \ldots, p_{n}^{\delta_{n}} \vdash A_{1}^{\sigma_{1}} \\
& p_{1}^{\delta_{1}}, p_{2}^{\delta_{2}}, \ldots, p_{n}^{\delta_{n}} \vdash A_{2}^{\sigma_{2}}
\end{aligned}
$$

Use one of the axiom schemes 4. - 6. we have

And for $A_{1}=\sigma_{1}, A_{2}=\sigma_{2}$

$$
p_{1}^{\delta_{1}}, p_{2}^{\delta_{2}}, \ldots, p_{n}^{\delta_{n}} \vdash A_{1}^{\sigma_{1}} \supset\left(A_{2}^{\sigma_{2}} \supset\left(A_{1} * A_{2}\right)^{\varphi_{*}\left(A_{1}, A_{2}, \sigma_{1}, \sigma_{2}\right)}\right)
$$

$$
p_{1}^{\delta_{1}}, p_{2}^{\delta_{2}}, \ldots, p_{n}^{\delta_{n}} \vdash A_{1}^{\sigma_{1}} \supset\left(A_{2}^{\sigma_{2}} \supset\left(A_{1} * A_{2}\right)^{\varphi_{*}\left(A_{1}, A_{2}, \sigma_{1}, \sigma_{2}\right)}\right)
$$

And $\left(A_{1} * A_{2}\right)^{\varphi_{*}\left(A_{1}, A_{2}, \sigma_{1}, \sigma_{2}\right)}$ is derived after the double application of m.m.p.

For the case 2. $A_{1}(\tilde{\delta})=\sigma_{1}, A_{2}(\tilde{\delta})=\sigma_{2}=>A(\tilde{\delta})=\left(A_{1}{ }^{A_{2}}\right)^{\left(\sigma_{1}{ }^{\sigma_{2}}\right)}$ and we must use the axiom scheme 7.

For the case 3. $A_{1}(\tilde{\delta})=\sigma \Rightarrow A(\tilde{\delta})=\neg \sigma$ we must use the axiom scheme 3 .

Corollary 1: If $A$ is a 3-tautology, then for every $\tilde{\delta}=\left(\delta_{1}, \delta_{2}, \ldots, \delta_{n}\right) \in E_{3}^{n}$

$$
p_{1}^{\delta_{1}}, p_{2}^{\delta_{2}}, \ldots, p_{n}^{\delta_{n}} \vdash A
$$

Theorem 1: Any formula is derived in P3 iff it is a 3-tautology.

Proof: It is obvious that every formula, derived in $P 3$, is a 3-tautology.

Let $P=\left\{p_{1}, p_{2}, \ldots, p_{n}\right\} \quad(n \geq 1)$ be the set of all variables of any tautology $A$. For every $\tilde{\delta}=$ $\left(\delta_{1}, \delta_{2}, \ldots, \delta_{n}\right) \in E_{3}^{n}$ by the above corollary, we have $p_{1}^{\delta_{1}}, p_{2}^{\delta_{2}}, \ldots, p_{n}^{\delta_{n}} \vdash A$.

For every $\delta_{1}, \delta_{2}, \ldots, \delta_{n-1}$ we take into consideration the following 3 truth values

$$
\left\{\begin{array}{l}
\delta_{1}, \delta_{2}, \ldots, \delta_{n-1}, 0 \\
\delta_{1}, \delta_{2}, \ldots, \delta_{n-1}, 1 / 2, \text { for which we have } \\
\delta_{1}, \delta_{2}, \ldots, \delta_{n-1}, 1
\end{array}\right.
$$

$$
\left\{\begin{array}{c}
p_{1}^{\delta_{1}}, p_{2}^{\delta_{2}}, \ldots, p_{n-1}^{\delta_{n-1}}, p_{n}^{0} \vdash A \\
p_{1}^{\delta_{1}}, p_{2}^{\delta_{2}}, \ldots, p_{n-1}^{\delta_{n-1}}, p_{n}^{1 / 2} \vdash A \\
p_{1}^{\delta_{1}}, p_{2}^{\delta_{2}}, \ldots, p_{n-1}^{\delta_{n-1}}, p_{n}^{1} \vdash A
\end{array}\right.
$$

By deduction theorem we have

$$
\begin{gathered}
p_{1}^{\delta_{1}}, p_{2}^{\delta_{2}}, \ldots, p_{n-1}^{\delta_{n-1}} \vdash p_{n}^{0} \supset A \\
p_{1}^{\delta_{1}}, p_{2}^{\delta_{2}}, \ldots, p_{n-1}^{\delta_{n-1}} \vdash p_{n}^{1 / 2} \supset A \\
p_{1}^{\delta_{1}}, p_{2}^{\delta_{2}}, \ldots, p_{n-1}^{\delta_{n-1}} \vdash p_{n}^{1} \supset A
\end{gathered}
$$

Then

$$
\begin{aligned}
& p_{1}^{\delta_{1}}, p_{2}^{\delta_{2}}, \ldots, p_{n-1}^{\delta_{n-1}} \vdash\left(A^{1} \supset B\right) \supset\left(\left(A^{1 / 2} \supset B\right) \supset\left(\left(A^{0} \supset B\right) \supset B\right)\right) / \text { axiom 8./ } \\
& p_{1}^{\delta_{1}}, p_{2}^{\delta_{2}}, \ldots, p_{n-1}^{\delta_{n-1}} \vdash\left(p_{n}^{1 / 2} \supset A\right) \supset\left(\left(p_{n}^{0} \supset A\right) \supset A\right) / \text { m.m.p./ } \\
& p_{1}^{\delta_{1}}, p_{2}^{\delta_{2}}, \ldots, p_{n-1}^{\delta_{n-1}} \vdash\left(p_{n}^{0} \supset A\right) \supset A / \text { m.m.p./ } \\
& p_{1}^{\delta_{1}}, p_{2}^{\delta_{2}}, \ldots, p_{n-1}^{\delta_{n-1}} \vdash A \text { /m.m.p./ }
\end{aligned}
$$


So, the number of premises is now $n-1$. Repeating the above steps, we finally obtain the derivation of tautology $A$ in $P 3$

Note that this proof is the complete analogy to proof of the corresponding theorem for the 2valued logic [2]. Also note that after proving by analogy the corresponding Lemma 1 . for any $k$ valued logic for $k \geq 4$, the proof of the corresponding theorem above can be given by analogy as well.

Every such PPS can be easily transformed into Gentzen-like system as well.

\section{Examples of Uniform PPS for Some Versions of MVL}

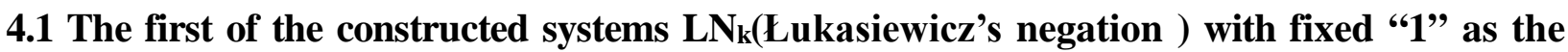
designated value, uses conjunction, disjunction, (1) implication, (1) negation and (1) exponent, as well as constants $\delta=\frac{i}{k-1}(1 \leq i \leq k-2)$ for using (1) exponent [3,5,7].

In particular, for all formulas $A, B, C$ of 3 -valued logic and each $\sigma_{1}, \sigma_{2}$ from the set $\{0,1 / 2,1\}$, the following formulas are axiom schemes of $\boldsymbol{L N}_{3}$.

1. $A \supset(B \supset A)$

2. $(A \supset B) \supset((A \supset(B \supset C)) \supset(A \supset C))$

3. $A^{\sigma_{1}} \supset\left(B^{\sigma_{2}} \supset(A \supset B)^{\sigma_{1} \supset \sigma_{2}}\right)$

4. $A^{\sigma_{1}} \supset\left(B^{\sigma_{2}} \supset(A \vee B)^{\sigma_{1} \vee \sigma_{2}}\right)$

5. $A^{\sigma_{1}} \supset\left(B^{\sigma_{2}} \supset(A \& B)^{\sigma_{1} \& \sigma_{2}}\right)$

6. $\left.A^{\sigma} \supset(\neg A)\right\urcorner \sigma$

7. $\left(A^{1} \supset B\right) \supset\left(\left(A^{1 / 2} \supset B\right) \supset\left(\left(A^{0} \supset B\right) \supset B\right)\right)$

Inference rule is modus ponens /m.p. $/ \frac{A, A \supset B}{B}$.

4.2. The second systems $\mathrm{CN}_{\mathrm{k}}$ (cyclically permuting negation) with fixed " 1 " as the designated value, use conjunction, disjunction, (2) implication, (2) negation and (2) exponent $[4,6,7]$.

In particular, for all formulas $A, B, C$ of 3 -valued logic and each $\sigma_{1}, \sigma_{2}$ from the set $\{0,1 / 2,1$, the following formulas are axiom schemes of $\mathrm{CN}_{3}$

1. $A \supset(B \supset A)$

2. $(A \supset B) \supset((A \supset(B \supset C)) \supset(A \supset C))$

3. $A^{\sigma_{1}} \supset\left(B^{\sigma_{2}} \supset(A \supset B)^{\varphi_{\supset}\left(A, B, \sigma_{1}, \sigma_{2}\right)}\right)$

4. $A^{\sigma_{1}} \supset\left(B^{\sigma_{2}} \supset(A \vee B)^{\varphi_{\vee}\left(A, B, \sigma_{1}, \sigma_{2}\right)}\right)$

5. $A^{\sigma_{1}} \supset\left(B^{\sigma_{2}} \supset(A \& B)^{\varphi_{\&}\left(A, B, \sigma_{1}, \sigma_{2}\right)}\right)$

6. $A^{\sigma_{1}} \supset\left(B^{\sigma_{2}} \supset\left(A^{B}\right)^{\varphi_{\exp }\left(A, B, \sigma_{1}, \sigma_{2}\right)}\right)$

7. $A^{\sigma} \supset(\neg A)^{\bar{\sigma}}$

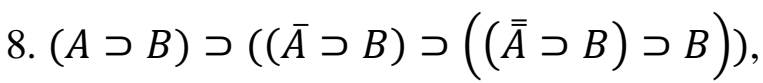

where the main role is played by the following exponents: 


$$
\begin{gathered}
\varphi_{\supset}\left(A, B, \sigma_{1}, \sigma_{2}\right)=\left(\sigma_{1} \supset \sigma_{2}\right) \&(\neg(A \bigvee \bar{A}) \bigvee(\overline{\bar{B}} \supset B)) \bigvee(\neg(A \bigvee \overline{\bar{A}}) \& \neg(B \bigvee \overline{\bar{B}})), \\
\varphi_{\vee}\left(A, B, \sigma_{1}, \sigma_{2}\right)=\left(\sigma_{1} \bigvee \sigma_{2}\right) \bigvee((A \supset \bar{A}) \& \neg(\bar{B} \bigvee \overline{\bar{B}})) \mathrm{V}(\neg(\bar{A} \bigvee \overline{\bar{A}}) \&(B \supset \bar{B})), \\
\varphi_{\&}\left(A, B, \sigma_{1}, \sigma_{2}\right)=\left(\sigma_{1} \& \sigma_{2}\right) \mathrm{V}((A \& \overline{\bar{A}}) \mathrm{V}(B \& \bar{B})) \mathrm{V}((A \& \bar{A}) \mathrm{V}(B \& \overline{\bar{B}}) \\
\varphi_{\exp }\left(A, B, \sigma_{1}, \sigma_{2}\right)=\sigma_{1}{ }^{\sigma_{2}} \bigvee\left(\neg\left(\sigma_{1}^{\sigma_{2}}\right) \& \neg\left(\neg\left(A^{\sigma_{1}} \& \bar{B}^{\sigma_{2}}\right) \bigvee \neg \neg\left(A^{\sigma_{1}} \& \bar{B}^{\sigma_{2}}\right)\right)\right)
\end{gathered}
$$

Inference rule is modus ponens /m.p. $/ \frac{A, A \supset B}{B}$.

4.3. For $L N_{3,2}$ - Lukasiewicz's logic with fixed " $1 / 2$ " and "1" as the designated value, which use conjunction, disjunction, (1) implication, (1) negation and (1) exponent, as well as constants $0,1 / 2$ and 1 for using (1)exponent.

In particular for all formulas A, B, C of 3-valued logic and each $\sigma_{1}, \sigma_{2}$ from the set $\{0,1 / 2,1\}$, the following formulas are axiom schemes of $\mathrm{LN}_{3,2}$ :

1. $A \supset(B \supset A)$

2. $(A \supset B) \supset\left((A \supset(B \supset C)) \supset\left(A \supset C^{1 / 2}\right)\right)$

3. $A^{\sigma_{1}} \supset\left(B^{\sigma_{2}} \supset(A \supset B)^{\varphi_{\supset}\left(A, B, \sigma_{1}, \sigma_{2}\right)}\right)$

4. $A^{\sigma_{1}} \supset\left(B^{\sigma_{2}} \supset(A \vee B)^{\varphi_{\vee}\left(A, B, \sigma_{1}, \sigma_{2}\right)}\right)$

5. $A^{\sigma_{1}} \supset\left(B^{\sigma_{2}} \supset(A \& B)^{\varphi_{\&}\left(A, B, \sigma_{1}, \sigma_{2}\right)}\right)$

6. $A^{\sigma_{1}} \supset\left(B^{\sigma_{2}} \supset\left(A^{B}\right)^{\varphi_{\exp }\left(A, B, \sigma_{1}, \sigma_{2}\right)}\right)$

7. $A^{\sigma} \supset(\neg A)^{\bar{\sigma}}$

8. $\left(A^{1} \supset B\right) \supset\left(\left(A^{1 / 2} \supset B\right) \supset\left(\left(A^{0} \supset B\right) \supset B\right)\right)$, where

$\varphi_{\supset}\left(A, B, \sigma_{1}, \sigma_{2}\right)=\bar{A} \bigvee B \bigvee \overline{\sigma_{1}} \bigvee \sigma_{2}$

$\varphi_{\bigvee}\left(A, B, \sigma_{1}, \sigma_{2}\right)=A \bigvee B \bigvee \sigma_{1} \bigvee \sigma_{2}$

$\varphi_{\&}\left(A, B, \sigma_{1}, \sigma_{2}\right)=\left(A \bigvee \sigma_{1} \bigvee \overline{B^{\sigma_{2}}}\right) \&\left(B \bigvee \sigma_{2} \bigvee \overline{A^{\sigma_{1}}}\right)$

$\varphi_{\text {exp }}\left(A, B, \sigma_{1}, \sigma_{2}\right)=A^{B} \bigvee \sigma_{1}{ }^{\sigma_{2}}$

$\varphi_{-}\left(A, B, \sigma_{1}, \sigma_{2}\right)=\bar{A} \bigvee \overline{\sigma_{1}}$

and inference rule is modification of modus ponens $\frac{A, A \supset B}{B^{\varphi_{m} .(A, B)}}$, where $\varphi_{m . p .}(A, B)=1 / 2$.

The work with another version of MVL and for $k=4$ or $k=8$ is in progress.

\section{Conclusion}

We suggest some Hilbert-like propositional proof system, which is universal for all versions of many-valued logic. Here we give also the application of the suggested system to 3 versions of 3valued logic, two of which have only one designated value and the last one has two designated values. The investigation of the applications to the other versions of $k$-valued logics $(k \geq 4)$ is in progress. 


\section{Acknowledgements}

This work was supported by the RA MES State Committee of Science, in the frames of the research project № 18T-1B034.

I am grateful to my supervisor, Professor of YSU Anahit Chubaryan for her encouragement and fruitful discussions, and also for helpful suggestions, which enabled to improve and expand the results.

\section{References}

[1] J. Lukasiewicz, “O Logice Trójwartósciowej”, Ruch Filozeficzny English translation: On three valued-logic, vol. 5, pp. 169-171, 1920.

[2] E. Mendelson, Introduction to Mathematical Logic, Van Nostrand, Princeton, 1975.

[3] A. A. Chubaryan, A. S. Tshitoyan and A. A. Khamisyan, "On some proof systems for many-valued logics and on proof complexities in it", (in Russian) Reports of NASA RA, vol. 116, no. 2, pp. 18-24, 2016.

[4] A. Chubaryan and A. Khamisyan, "Generalization of Kalmar's proof of deducibility in two valued propositional logic into many valued logic", Pure and Applied Mathematics Journal, doi: 10.116448/j.pamj. 20170602.12, vol. 6, no. 2, pp. 71-75, 2017.

[5] A. Chubaryan, A. Khamisyan and A. Tshitoyan, "On some systems for Łukasiewicz's many-valued logic and its properties”,FundamentalisScientiam, vol. 8, no. 8, Spain, pp. 74-79, 2017.

[6] А. Чубарян и А. Хамисян, Новый метод доказательства полноты пропозициональной системы трехзначной логики Лукасевича и его приложения, Evolutio, Естественные науки, Вып. 3, сс. 9-12, 2016.

[7] A.Chubaryan, A.Khamisyan and G. Petrosyan, On Some Systems for Two Versions of Many-valued Logics and its Properties, Lambert Academic Publishing (LAP), 2017.

Submitted 18.12.2019, accepted 09.03.2020. 


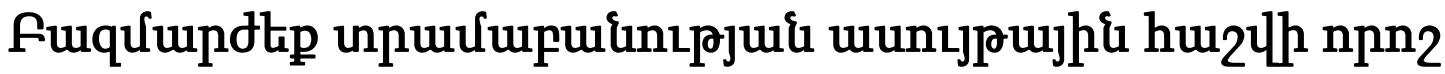

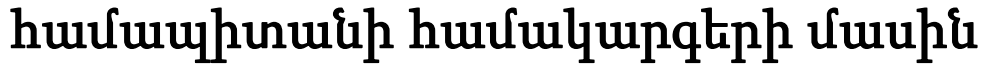

\author{
Unpnıp U. Fưưhujui \\ Eplumih ütunulumi hưưuuupuis \\ e-mail: khamisyam@gmail.com

\section{Uựnnuntu}

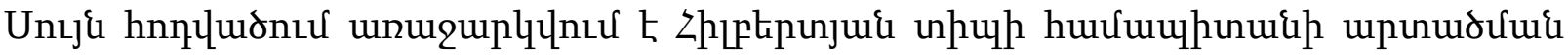

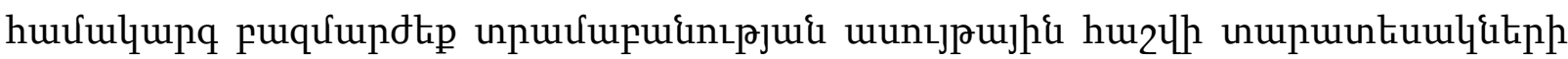

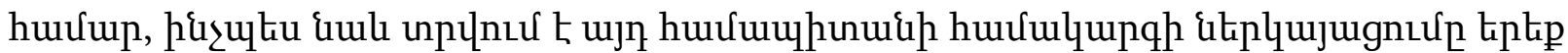

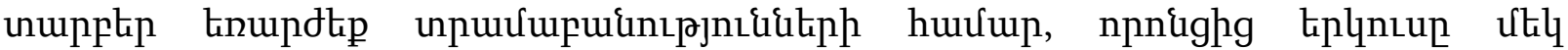

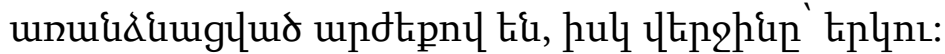

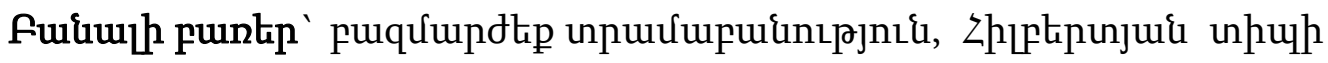

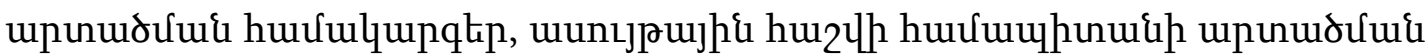
huruulquipq:

\section{О некоторых универсальных пропозициональных системах ВЫВодов для многозначных логик}

\author{
Артур А. Хамисян \\ Ереванский государственный университет \\ e-mail:khamisyam@gmail.com
}

\begin{abstract}
Аннотация
В данной статье предлагается некоторая универсальная пропозициональная система Гильбертовского типа для всех версий многозначных логик и рассматриваются ее приложения для трех версий 3-значных логик, две из которых имеют одно выделенное значение, а последняя - два выделенных значения.

Ключевые слова: многозначная логика, системы выводов Гильбертовского типа, универсальная пропозициональная система выводов.
\end{abstract}

